ARTICLE

Received 12 Nov 2013 | Accepted 13 Jan 2014 | Published 12 Feb $2014 \quad$ DOl: 10.1038/ncomms4249

\title{
High photoresponsivity in an all-graphene $p-n$ vertical junction photodetector
}

Chang Oh Kim ${ }^{1, \star}$, Sung Kim ${ }^{1, \star}$, Dong Hee Shin ${ }^{1}$, Soo Seok Kang ${ }^{1}$, Jong Min Kim¹, Chan Wook Jang ${ }^{1}$, Soong Sin Joo', Jae Sung Lee ${ }^{1}$, Ju Hwan Kim¹, Suk-Ho Choi ${ }^{1} \&$ Euyheon Hwang ${ }^{2}$

Intensive studies have recently been performed on graphene-based photodetectors, but most of them are based on field effect transistor structures containing mechanically exfoliated graphene, not suitable for practical large-scale device applications. Here we report highefficient photodetector behaviours of chemical vapor deposition grown all-graphene $p-n$ vertical-type tunnelling diodes. The observed photodetector characteristics well follow what are expected from its band structure and the tunnelling of current through the interlayer between the metallic $p$ - and $n$-graphene layers. High detectivity $\left(\sim 10^{12} \mathrm{~cm} \mathrm{~Hz}^{1 / 2} \mathrm{~W}^{-1}\right)$ and responsivity $\left(0.4 \sim 1.0 \mathrm{AW}^{-1}\right)$ are achieved in the broad spectral range from ultraviolet to near-infrared and the photoresponse is almost consistent under 6-month operations. The high photodetector performance of the graphene $p-n$ vertical diodes can be understood by the high photocurrent gain and the carrier multiplication arising from impact ionization in graphene.

\footnotetext{
${ }^{1}$ Department of Applied Physics, College of Applied Science, Kyung Hee University, Yongin 446-701, Korea. ${ }^{2}$ SKKU Advanced Institute of Nanotechnology, Sungkyunkwan University, Suwon 440-746, Korea. * These authors contributed equally to this work. Correspondence and requests for materials should be addressed to S.-H.C. (email: sukho@khu.ac.kr).
} 
P hotodetectors (PDs) are nowadays recognized not only as a single-function device in detecting optical signals but also as a key component in the optoelectronic integration circuits that consist of light emitters, optical waveguides, PDs and electronic components on a single chip ${ }^{1,2}$. As major commercial products, GaN-, Si- and InGaAs-based PDs are available in the three important, UV, visible, and near-IR (NIR) subbands, respectively. Contrary to other two counterparts operated at room temperature (RT), InGaAs PDs should be cooled at $4.2 \mathrm{~K}$ for high detectivity. It would be advantageous to have a low-cost multicolour PD system showing high performance at RT over the broad spectral range from UV to NIR. Graphene has offered a broad spectral bandwidth potentially up to $\sim 500 \mathrm{GHz}$ and fast response time down to $\sim 1.5 \mathrm{ps}$ in PD applications ${ }^{3-9}$, but weak light absorption and low gain have limited key figures of merit such as quantum efficiency $(\mathrm{QE})$, responsivity and noise equivalent power (NEP) of graphene PDs below $30 \%, 0.1 \mathrm{~A} \mathrm{~W}^{-1}$ and $200 n \mathrm{~W} \mathrm{~Hz}^{-1 / 2}$, respectively ${ }^{3,10,11}$. Commercially available PDs show much better performances, but are not useful for transparent and flexible electronics and photonics. Thus, the demonstration of photodetection gain with graphene would be the basis for a variety of applications in flexible and transparent optoelectronics for biomedical imaging, remote sensing and optical communications.

Previous studies of photocurrent (PC) in graphene have demonstrated photoresponse near metallic contacts $3,6-8$, at the interface between single-layer and bilayer regions ${ }^{12}$, at lateraltype $p-n$ junctions $s^{5,13,14}$, or in the heterostructures with twodimensional semiconductors ${ }^{10}$. The PC response was attributed to the presence of potential barriers at the metal contacts/the $p-n$ junctions or across the heterostructures and could be modulated by sweeping gate voltages ${ }^{3,4,6,10,13}$. However, all these studies were based on field effect transistor structures containing mechanically exfoliated graphene, not suitable for practical large-scale device applications, and the magnitudes of PC were shown to be typically very weak compared with those in conventional semiconductor PDs owing to the low QE of graphene.

Here we report a very practical chemical vapor deposition (CVD) graphene-based structure that works as a PD showing high performances in the UV-visible-NIR range. The PD structures permit a large PC flow by the tunnelling of charge carriers through the interlayer formed between the $p$ - and $n$ graphene layers at higher $n$-doping concentrations $s^{15}$.

\section{Results}

Dark and photo $I-V$ characteristics. We prepared graphene $p-n$ vertical tunnelling junctions for various doping concentrations of the $n$-type graphene layer by varying doping time $\left(t_{\mathrm{D}}\right)$ in the benzyl viologen (BV) exposure while $t_{\mathrm{D}}$ was fixed at $5 \mathrm{~min}$ in the $\mathrm{AuCl}_{3}$ exposure. The devices were named as D1 $\sim \mathrm{D} 5$ when $t_{\mathrm{D}}$ in the $\mathrm{BV}$ exposure was $0.5,1,2,3$ and $4 \mathrm{~min}$, respectively. The $t_{\mathrm{D}}$-dependent properties of the $n$ - and $p$-graphene layers were analysed in detail ${ }^{15}$ by using various structural, optical and electrical detection techniques ${ }^{16}$. Figure 1a shows a schematic of a typical graphene $p-n$ vertical tunnelling junction for photodetection. Positive voltages were applied to the $\mathrm{Ag}$ electrode on $p$-graphene layer under forward bias. The dark current (DC)-voltage $(I-V)$ curves are symmetric and linear in the forward/reverse directions with respect to zero voltage for D1-D3 devices, indicating no rectifying behaviours at the $p-n$

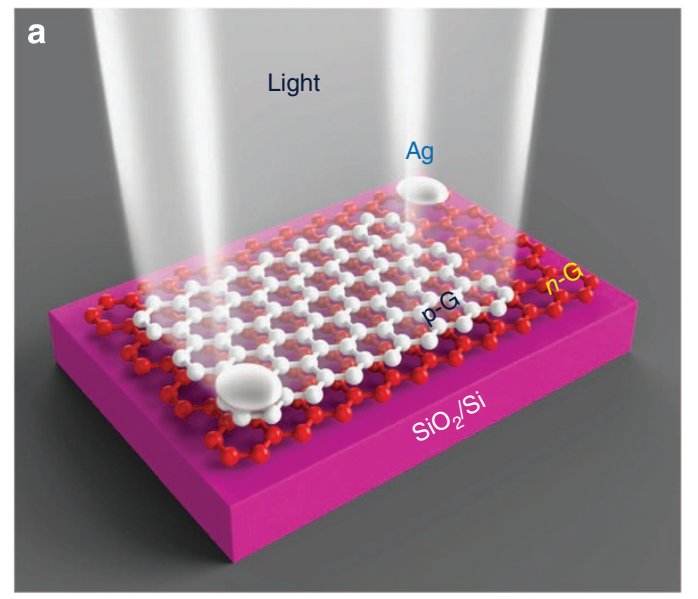

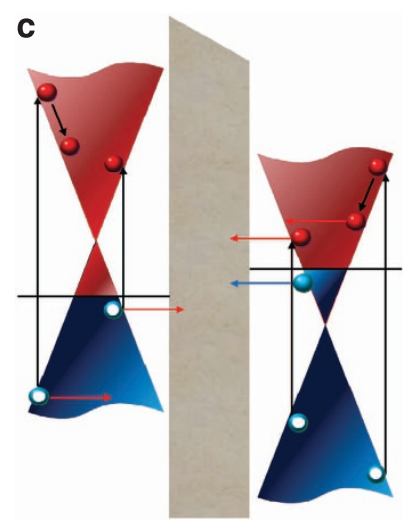

$V>0$

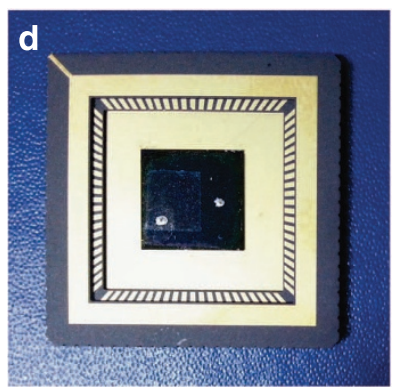

Figure 1 | Diagrams of schematics and band structure describing the PDs. Schematics of (a) a typical graphene $p-n$ diode for photodetection and (b) its magnified version showing an interlayer between metallic $p$ - and $n$-graphene ( $p$ - $G$ and $n$ - $G$ ) layers. The diodes are illuminated with defocused light sources (xenon lamp or laser) with spot sizes that well match the device window of $5 \times 5 \mathrm{~mm}^{2}$ size. For the electrodes, Ag was used. (c) Band diagram of the $p-n$ PD under forward (positive) bias. Red and white spots represent photoexcited electrons and holes, respectively, and lateral arrows indicate the transport directions of electrons and holes contributing to DCs and photocurrents. (d) A real image of a typical PD. 
junctions because of the negligible barrier at the boundary ${ }^{15}$. The $I-V$ characteristics of these devices are almost not varied even under illumination at various photon wavelengths from 300 to $1,000 \mathrm{~nm}$, as shown in Fig. 2a, resulting in no PC.

For $t_{\mathrm{D}}$ above $2 \mathrm{~min}$ (D4 and D5 devices), the DC is greatly reduced over the full range of bias voltage, with the current reduction being stronger under forward bias than under reverse bias, as shown in Fig. 2b (and Supplementary Fig. 1). The dark $I-V$ curves show nonlinear properties with varying bias voltage, indicating rectifying behaviours. These anomalous $I-V$ properties were attributed to actual formation of a structure like metalinsulator-metal/metal-semiconductor-metal diode due to strongly corrugated insulating or semiconducting interlayers formed between the metallic $p$ - and $n$-graphene sheets at higher $n$-doping concentrations (see Fig. $1 \mathrm{~b}$ ) and well explained based on the band structures of biased $p-n$ junctions ${ }^{15}$ (Supplementary Fig. 2). The DC is of the order of $10^{-8} \mathrm{~A}$ at a voltage of $\sim 5 \mathrm{~V}$ for D4 device, indicating a very low leakage. Under illumination, large PC is obtained for forward bias, as shown in Fig. 2b, indicating a very high photosensitivity in this very simple structure. The photoresponse strongly depends on the photon wavelength. We find that the PC is of the order of $\mu \mathrm{A}$ for the bias voltages $|V|>3 \mathrm{~V}$, as shown in Fig. $2 \mathrm{~b}$, and observe a negligible change in the total current between before and after light illumination for negative bias. Since the total current is given by $(\mathrm{DC}+\mathrm{PC})$ and the contribution of PC to the total current is negligible, that is, $\mathrm{DC} \gg \mathrm{PC}$ for reverse bias, the total current is mainly determined by $\mathrm{DC}$ for negative bias, thereby making PC invisible for reverse bias. The D5 device shows lower photosensitivity owing to the higher DC (Supplementary Fig. 1). The ratio of $\mathrm{PC}$ to $\mathrm{DC}$ or the on/off ratio of $\mathrm{D} 4$ device shows maximum intensities at bias voltages of around $2 \sim 3 \mathrm{~V}$ for various wavelengths $(\lambda)$, as shown in Fig. $2 \mathrm{c}$. The on/off ratio of D5 device shows similar bias voltage and $\lambda$ dependences, but its magnitude is much smaller, regardless of bias voltage and $\lambda$ (Supplementary Fig. 1), resulting from larger DC. These $I-V$ characteristics under dark as well as under

a
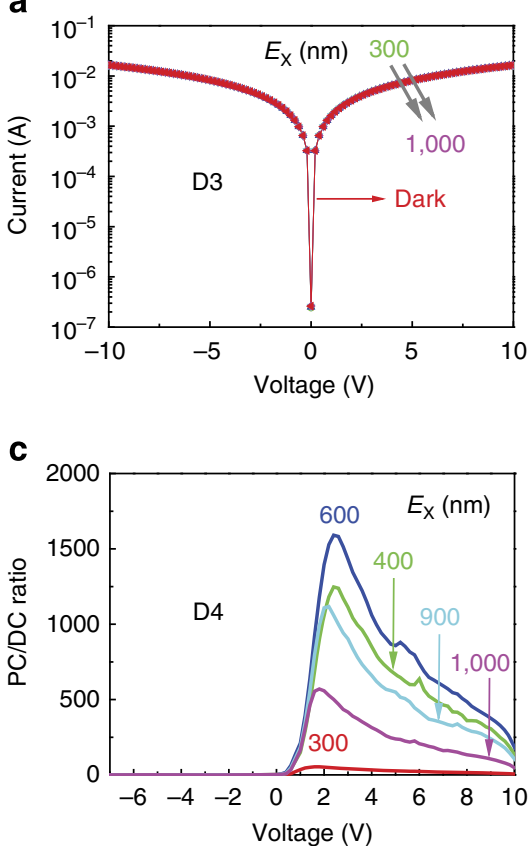

illumination were almost not varied by using $\mathrm{Au}$ as an electrode (Supplementary Fig. 3).

The rectifying behaviour of the DC can be understood from the available density of states for tunnelling between $p$ - and $n$-type graphene $^{15}$ (Supplementary Fig. 2). For positive bias the current is severely suppressed due to minimizing of joint density of states for tunnelling. When the graphene $p-n$ junction is illuminated by laser light, electrons in valence band are excited to conduction band, producing electron-hole pairs. In the presence of photoexcitation both electrons and holes contribute to the PC. Especially, in our graphene $p-n$ junction the tunnelling current by holes is more significant than the current by electrons for a positive bias because the tunnelling of electrons is restricted by the joint density of states, but there is no restriction for hole tunnelling, as shown in Fig. 1c. In graphene, since the relaxation due to the carrier-phonon scattering is much slower than that due to the carrier-carrier scattering ${ }^{17,18}$, a single photon produces multiple electron-hole excitations by impact ionization induced by a hot electron ${ }^{19,20}$. Thus, the carrier multiplication enhances the PC. The PC dominates over the DC when the DC is small for positive bias while the $\mathrm{PC}$ is not visible when the $\mathrm{DC}$ is very large for reverse bias, as shown in Fig. $2 \mathrm{~b}$ and Supplementary Fig. 1.

Photosensitivity and detectivity. We also prepared graphene $p-n$ vertical tunnelling junctions for various doping concentrations of the $p$-type graphene layer by varying $t_{\mathrm{D}}$ in the $\mathrm{AuCl}_{3}$ exposure while $t_{\mathrm{D}}$ was fixed at $3 \mathrm{~min}$ in the $\mathrm{BV}$ exposure, and found that the device characteristics were optimized at $t_{\mathrm{D}}=5$ and $3 \mathrm{~min}$ for $\mathrm{AuCl}_{3}$ and $\mathrm{BV}$ exposures (D4 device), respectively (Supplementary Fig. 3). We characterized D4 and D5 devices in detail by measuring several figures of merit essential for the evaluation of a PD. Figure $2 \mathrm{~d}$ shows responsivity (the electrical current response to the incident optical power, $R_{i}$ ) of $\mathrm{D} 4$ device, measured as a function of bias voltage for various $\lambda$. There are no big variations in $R_{i}$ above around $2 \sim 3 \mathrm{~V}$ irrespective of $\lambda . R_{i}$ at $\lambda \sim 900 \mathrm{~nm}$ reaches $\sim 0.7 \mathrm{~A} \mathrm{~W}^{-1}$ at $6 \mathrm{~V}$. At $\lambda \sim 400 \mathrm{~nm}, R_{i}$ is

b

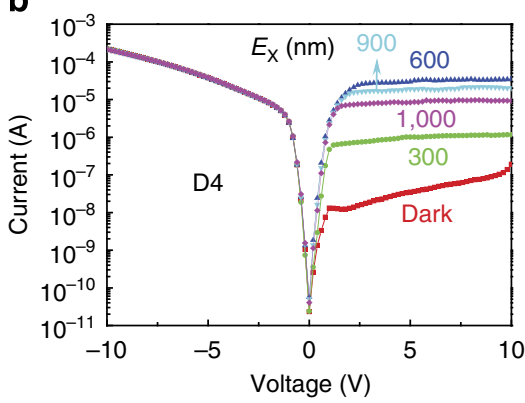

d

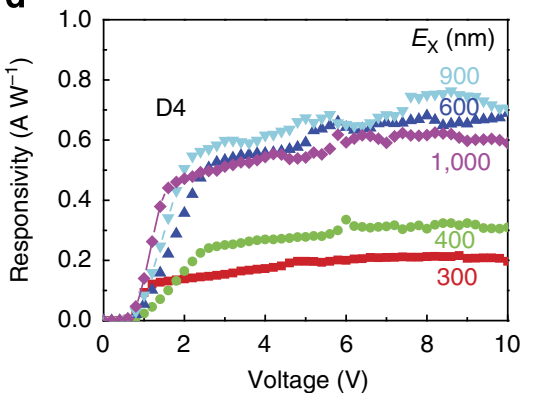

Figure 2 | Dark and photo I-V characteristics and bias-dependent responsivity. I-V characteristics of (a) D3 and (b) D4 devices under dark and illumination at various photon wavelengths from 300 to 1,000 nm. (c) On/off current ratios of D4 device as functions of bias voltage at various photon wavelengths from 300 to $1,000 \mathrm{~nm}$. (d) Responsivities of D4 device as functions of forward bias at various photon wavelengths from 300 to 1,000 nm. 
reduced to $\sim 0.3 \mathrm{~A} \mathrm{~W}^{-1}$ because less light was absorbed by the device. Figure 3a,b compares the spectral $R_{i}$ of D4 and D5 devices for bias voltages from 1 to $5 \mathrm{~V}$. There are small variations in the spectral $R_{i}$ of $\mathrm{D} 4$ device for bias voltages $\geq 2 \mathrm{~V}$. In contrast, the D5 shows a gradual increase in the spectral $R_{i}$ with increasing the bias voltage up $5 \mathrm{~V}$; in other words, the $R_{i}$ is more sensitive to the variation of the bias voltage in D5 device (Supplementary Fig. 1). The $R_{i}$ of D5 device in the UV range $(300-400 \mathrm{~nm})$ reaches $\sim 0.4 \mathrm{~A} \mathrm{~W}^{-1}$ at $6 \mathrm{~V}$, (Supplementary Fig. 1) while that in the NIR range $(700-950 \mathrm{~nm})$ is as high as $\sim 1.0 \mathrm{~A} \mathrm{~W}^{-1}$, as shown in Fig. 3b. These $R_{i}$ values are higher than (or comparable to) those reported for commercially available $\mathrm{Si}$ and InGaAs PDs $(\sim 0.5$ and $\sim 0.9 \mathrm{~A} \mathrm{~W}^{-1}$, respectively $)^{21,22}$. The DC decreases with decreasing temperature $(T)$, resulting in higher on/off ratio and $R_{i}$ under forward bias at lower $T$ (Supplementary Fig. 4).

The QE can be calculated by normalizing the number of photogenerated carriers by the number of absorbed photons for any given $\lambda$. In both D4 and D5 devices, the magnitude of the QE increases with increasing $V$; however, this increase is not linear (Supplementary Fig. 5). Furthermore, the QE is not spectrally uniform and shows a relatively clear peak around $\sim 500 \mathrm{~nm}$ in D4 device, but the QE peak is not pronounced in D5 device. By considering the spectral $R_{i}$ in Fig. 3a,b, it is noted that a strong enhancement is especially reflected in high-energy side of QE, consistent with the fact that the absorption spectra of doped graphene sheets are peaked at high energies (Supplementary Fig. 6). The QEs of D4 and D5 devices are over 100\% at bias voltages of 4 and $5 \mathrm{~V}$, which will be explained later in detail based on the transient PC data.

A key figure of merit for a PD is the NEP. This parameter is defined as the amount of incident light power that generates a PC equal to the noise current. The NEP can be expressed $a^{23}$ : $\mathrm{NEP}=\left(\left\langle I_{n}^{2}\right\rangle\right)^{1 / 2} / R_{i}$ where $\left\langle I_{n}^{2}\right\rangle$ is the time-averaged square of the total noise current and can be estimated by integrating
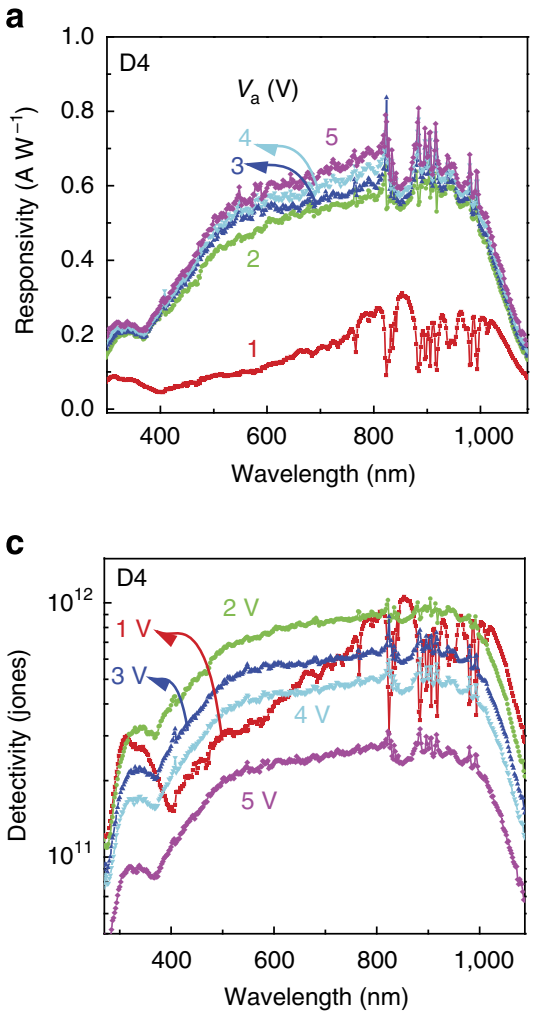

spectral density of noise power (Supplementary Fig. 7). Then, the NEP can be calculated by using the spectral $R_{i}$ data in Fig. 3a,b; (Supplementary Fig. 7). Another parameter used to characterize the performance of PDs is the detectivity $\left(D^{*}\right)$, which is related to NEP by the expression: $D^{*}=(A \Delta f)^{1 / 2} /$ NEP. Here $A$ is the effective area $(\sim 0.5 \times 0.5)$ of the detector in $\mathrm{cm}^{2}$ and $\Delta f$ is the electrical bandwidth in $\mathrm{Hz}$. The $D^{*}$ of both devices increases with increasing the bias up to $\sim 2 \mathrm{~V}$, but above $\sim 2 \mathrm{~V}$ it decreases over almost full range of $\lambda$, as shown in Fig. $3 \mathrm{c}$,d. The maximum $D^{*}$ at $\sim 2 \mathrm{~V}$ is closely related to the largest on/off ratio at $2 \sim 3 \mathrm{~V}$, as shown in Fig. 2c. Relatively poor $D^{\star}$ for D5 device may be due to high DC (Supplementary Fig. 1). Owing to its fairly 'flat' spectrum, the $D^{\star}$ of D4 device remains high on both the blue $\left(4.0 \times 10^{11} \mathrm{~cm} \mathrm{~Hz}^{1 / 2} \mathrm{~W}^{-1}\right.$ or Jones at $\left.400 \mathrm{~nm}\right)$ and the NIR $\left(1.0 \times 10^{12}\right.$ Jones at $\left.900 \mathrm{~nm}\right)$ ends of the spectrum at $2 \mathrm{~V}$. The $D^{*}$ value of D5 device (for example, $4.8 \times 10^{10}$ and $1.2 \times 10^{11}$ Jones at 400 and $900 \mathrm{~nm}$, respectively, for a bias voltage $=3 \mathrm{~V}$ ) is smaller than that of device D4, but the response of D5 device is more spectrally uniform for bias voltages $\geq 2 \mathrm{~V}$. These values are comparable to those of $\mathrm{Si}$ and InGaAs PDs $\left(\sim 4 \times 10^{12}\right.$ and $>10^{12}$ Jones $(4.2 \mathrm{~K})$, respectively $)^{24}$.

Linearity and time response. Figure $4 \mathrm{a}$ shows $\mathrm{PC}$ density $\left(J_{\mathrm{PC}}\right)$ of D4 device versus incident light power of a 532-nm laser line at various bias voltages, which was obtained at a modulation frequency of $30 \mathrm{~Hz}$. The $J_{\mathrm{PC}}$ strongly depends on the illumination power. Neutral density filters were used to change the incident light power $(P)$. For lower light powers spanning three orders of magnitude, the $J_{\mathrm{PC}}$ increases linearly with $P$, and after a threshold power it deflects from the linear relationship. The threshold power increases with increasing the bias voltage, as shown in Fig. 4a. The $J_{\mathrm{PC}}$ in the linear region can be expressed by a simple power law: $J_{\mathrm{PC}} \propto P^{\alpha}$, where $0.95<\alpha<1.08$ in this work. The linear
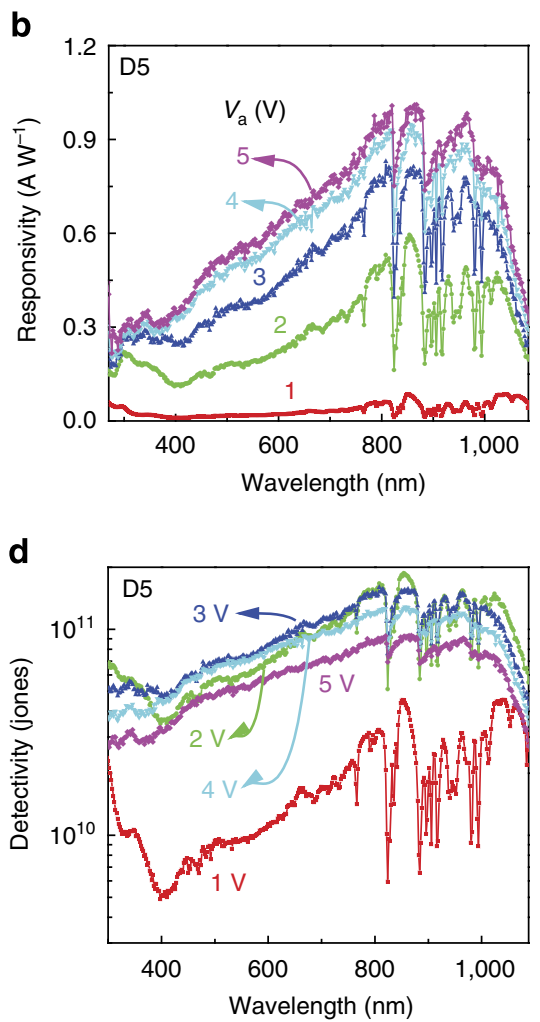

Figure 3 | Spectral responsivity and detectivity. Spectral responsivities of (a) D4 and (b) D5 devices at different forward-bias voltages from 1 to 5 V. Spectral detectivities of (c) D4 and (d) D5 devices at different forward-bias voltages from 1 to $5 \mathrm{~V}$. 
a

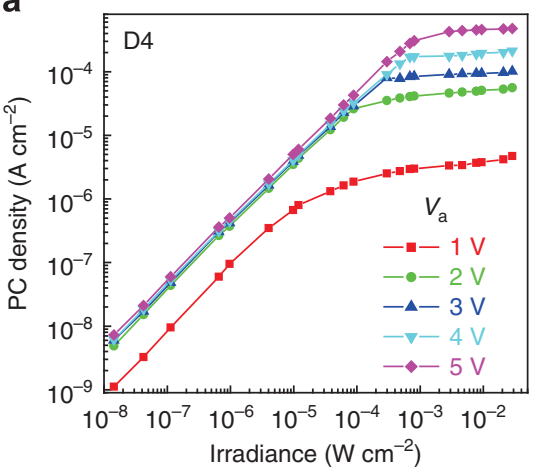

C

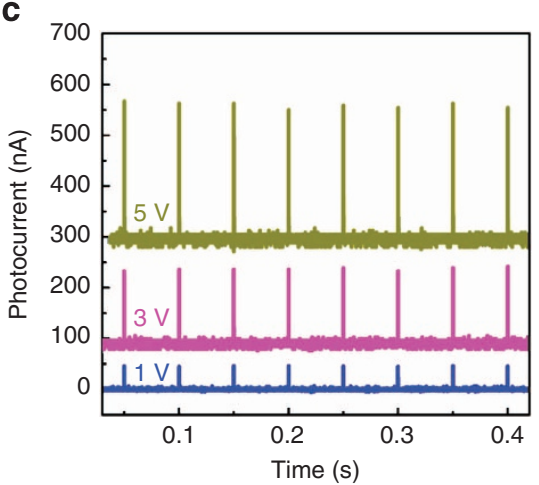

b

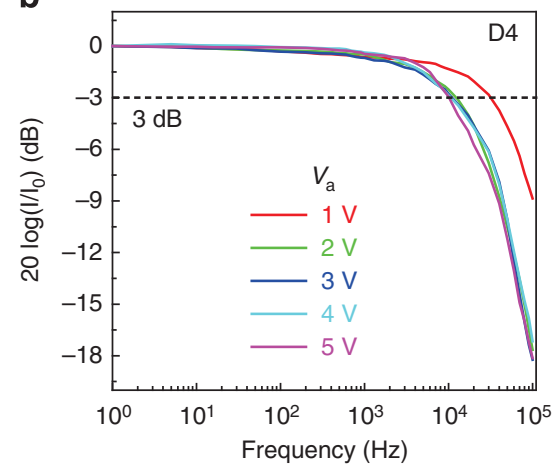

d

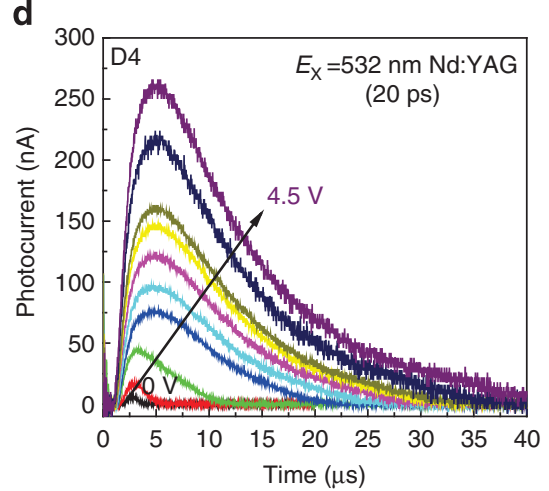

Figure 4 | Linearity and time response. (a) Irradiance-dependent PC densities of D4 device at different forward-bias voltages from 1 to 5 V. (b) Frequencydependent PC response of D4 device at different forward-bias voltages from 1 to $5 \mathrm{~V}$. (c) Repeated PC response switching of D4 device at different forward-bias voltages from 1 to $5 \mathrm{~V}$. (d) Transient PCs of D4 device at different forward-bias voltages from 0 to $4.5 \mathrm{~V}$ in $0.5 \mathrm{~V}$ step. The PC switching and transient behaviours were measured by using 20 ps, $20 \mathrm{~Hz} \mathrm{Nd:YAG} \mathrm{laser} \mathrm{pulses} \mathrm{at} 532 \mathrm{~nm}$.

increase of the PC density is attributed to the linear photogeneration of electron-hole pairs. However, at high powers the photogenerated electron-hole pairs can recombine before reaching electrodes possibly because of their trapping at the surface/interface states ${ }^{25}$, resulting in the saturation of the PC density at high powers.

Another figure of merit for PDs is the linear dynamic range (LDR) or photosensitivity linearity (typically quoted in $\mathrm{dB}$ ). LDR is given by $\mathrm{LDR}=20 \log \left(J^{*}{ }_{\mathrm{PC}} / J_{\mathrm{d}}\right)$, where $J^{*}{ }_{P C}$ is the PC density measured at a light intensity of $1 \mathrm{~mW} \mathrm{~cm}-2$ and $J_{\mathrm{d}}$ is the DC density. The ratio of full-well capacity of today's complementary metal-oxide-semiconductor image sensors to the number of dark electrons limits dynamic range to about three orders of magnitude in intensity (conventionally expressed as $60 \mathrm{~dB}$ in imaging); in other words, more than $60 \mathrm{~dB}$ is required for the imaging application of PDs. As calculated from the $J_{\mathrm{PC}}$ versus irradiance curve, as shown in Fig. 4 a, the LDR of D4 device is $\sim 93 \mathrm{~dB}$ ( $\sim 85 \mathrm{~dB}$ for D5 device; Supplementary Fig. 8 ), which is comparable to that of Si PDs $(\sim 120 \mathrm{~dB})$ and significantly higher than that of InGaAs PDs $(\sim 66 \mathrm{~dB})^{24,26}$. The LDR increases with increasing the bias voltage (Supplementary Fig. 8).

Figure $4 \mathrm{~b}$ shows the frequency $(f)-\mathrm{PC}$ response of $\mathrm{D} 4$ device. The PC response is independent of $f$ at $f<\sim 3 \times 10^{4} \mathrm{~Hz}$ at $1 \mathrm{~V}$, but begins to decrease at $f \geq \sim 3 \times 10^{4} \mathrm{~Hz}$. This transition corresponds to the minimum carrier lifetime required for photocarriers generated in the $n$ (or $p$ ) region to tunnel into the $p$ (or $n$ ) region. The 3- $\mathrm{dB}$ frequency decreases with increasing the forward bias, as shown in Fig. $4 \mathrm{~b}$ (Supplementary Fig. 8 for D5 device), because of an increase in tunnelling time with increasing electric field in the interlayer region between the $p$ - and $n$-graphene sheets. The linearity of $R_{i}$ with the light power is also enhanced at higher bias voltages (Supplementary Fig. 8).
Figure 4c shows PC response switching at various bias voltages by using $20 \mathrm{~Hz}, 532 \mathrm{~nm}$ laser pulse. The laser was focused onto the PD devices with a spot size of $\sim 5 \times 5 \mathrm{~mm}^{2}$. Within $40 \mu \mathrm{s}$ after the light is turned off, the current reaches the initial DC value. The PC switching behaviours are very regular for each pulse, almost irrespective of the bias voltage. The D5 device shows similar switching behaviours (Supplementary Fig. 9). To probe the charge-transfer dynamics, transient PCs were recorded at much smaller timescale for various bias voltages, as shown in Fig. 4d; (Supplementary Fig. 9 for D5 device). The turn-on transient response is characterized by a relatively fast increase in the PC during several tens of ns, followed by a relatively slow decay to a steady-state value during several $\mu \mathrm{s}$, which is attributed to electron-hole recombination. The rise time is the time required for generation of carriers in the $n$ or $p$ region and tunnelling in the $p-n$ junction, and is dependent on the width of the interlayer. As reported before ${ }^{27}$, the charge state of the BV molecules in the interlayer can be changed by UV illumination, thereby changing the film transmittance, but the response time is approximately several tens of seconds, much longer than the decay time in our work. Instead, considerable amount of trap states can be formed at the interlayer/p- and $n$-graphene sheets, thereby inducing multiple trappings of carriers, resulting in slower decay of photoexcited carriers in the graphene $p-n$ device, compared with the field effect transistor structures containing pristine graphene ${ }^{3,9}$. The decay time as well as the rise time increases with increasing the bias voltage for the D4 and D5 devices (Supplementary Fig. 9) but these behaviours do not seem to be relevant to the RC time constant of the $p-n$ structures, showing a decrease with the increase of the bias voltage (Supplementary Fig. 9). The switching time is estimated to be tens of $\mu$ s depending on the bias voltage. The PDs should be reliable under long-term 
operations. Therefore, we studied long-term stabilities of the PD performance by measuring $R_{i}$ at various voltages for 6 months in air, and found the $R_{i}$ was consistent under 6-month operations regardless of bias voltage (Supplementary Fig. 10).

\section{Discussion}

It should be noted that the measured QEs are the effective ones, that is, $\eta_{\text {eff }}=\eta G$, where $\eta$ and $G$ are the QE and the internal gain of the device, respectively ${ }^{28}$. We expect the D4 and D5 devices can show remarkably high $\eta$ values owing to a carrier multiplication of $\sim 2.5$ in graphene ${ }^{19}$, the doping-enhanced absorption reaching up to $\sim 10 \%$ in each $n$ - or $p$-graphene layer ${ }^{15}$ and the $p-n$ graphene double-layer structure. In addition, extremely high gain is expected in D4 and D5 devices because the gain is given by the ratio of the carrier lifetime $(\tau)$ to the transit time $(t)$ across the electrodes, $G \sim \tau / t$, and from the transient PC the lifetime of electron-hole pair is estimated to be an order of $\mu$ s (Supplementary Fig. 9). The electron-hole pairs are produced within several tens of ns (Supplementary Fig. 9) on a light pulse and in the vertical junction they transit to the opposite electrode immediately from the place where they are generated. Thus, the current gain in the vertical junction can exceed an order of $10^{2}$. Combining these two effects, it is possible to obtain effective QEs almost over 100\% in D4 and D5 devices. However, in the lateral graphene $p-n^{5,13,14}$ or the graphene-metal ${ }^{3,4}$ junctions the gain is expected to be very low because the spacing between the electrodes is typically an order of $\mu \mathrm{m}$. Thus, it may be possible to get the high effective QE only in vertical $p-n$ junctions.

These results demonstrate that the performance parameters of all-graphene $\mathrm{PDs}$ based on their $p-n$ vertical junctions are comparable to or even better than commercially available PDs made of $\mathrm{Si}$ or compound semiconductor materials. The achievement of high-performance all-graphene PDs implies that graphene can be used in a variety of possible transparent and flexible optoelectronics applications and will open exciting opportunities not only for the creation of single-function PDs with wide spectral range but also for the fabrication of high-resolution PD arrays for imaging, sensing and optical communications.

\section{Methods}

Doping of graphene. The graphene films were prepared by CVD and subsequently transferred to the $300-\mathrm{nm} \mathrm{SiO}_{2} / p$-type $\mathrm{Si}$ wafers. Gold chloride $\left(\mathrm{AuCl}_{3}\right)$ powder and 1, $1^{\prime}$-dibenzyl-4,4'-bipyridinium dichloride $\left(\mathrm{BV}^{2+}\right)$ (Sigma-Aldrich) were used for $p$ - and $n$-type dopants, respectively. $\mathrm{AuCl}_{3}$ powder was dissolved in nitromethane to yield $10 \mathrm{mM}$ solution and $\mathrm{BV}^{2}+$ was reduced in a biphasic solution of water/toluene to prepare $10 \mathrm{mM} \mathrm{BV}^{0}$ solution ${ }^{15}$. For the doping of graphene, the dopant solution was dropped on the whole surface of graphene sheet and after some time (this time was defined as doping time, $t_{\mathrm{D}}$ ) elapsed, it was spin coated at 2,000 r.p.m. for $1 \mathrm{~min}$. The $t_{\mathrm{D}}$ was varied to change doping concentration.

Fabrication of $\boldsymbol{p}-\boldsymbol{n}$ devices. For the formation of graphene $p-n \mathrm{PD}$, a solution of BV was first dropped and spin coated on the $10 \times 10-\mathrm{mm}^{2}$ graphene $/ \mathrm{SiO}_{2} /$ p-type Si wafer, and then annealed at $100^{\circ} \mathrm{C}$ for $10 \mathrm{~min}$ to make graphene uniformly $n$-type. The annealing temperature was optimized through repeated experiments for the good quality-doped graphene. Subsequently, a $5 \times 5-\mathrm{mm}^{2}$ bare graphene was transferred on $\sim 1 / 4$ area of the $n$-graphene/ $\mathrm{SiO}_{2} / p$-type $\mathrm{Si}$ wafer, a solution of $\mathrm{AuCl}_{3}$ was dropped and spin coated on the surface of graphene, and similarly annealed. As a result, the graphene $p-n$ vertical junction was formed on the $\sim 1 / 4$ area of the $\mathrm{SiO}_{2} / p$-type $\mathrm{Si}$ wafer. One millimetre diameter $\mathrm{Ag}$ or $\mathrm{Au}$ electrodes were deposited on the top of both $n$ - and $p$-graphene layers to complete the graphene $p-n$ PD.

Measurements. $I-V$ measurements to characterize the electrical behaviours of graphene $p-n$ PDs were carried out using a Keithley 2,400 source meter controlled by a LabView program. The DC noise under forward bias was measured with a dynamic signal analyser (Agilent 35670A) coupled to a low-noise current preamplifier (Stanford Research SR570) in the frequency range of $1 \mathrm{~Hz} \sim 5 \mathrm{kHz}$. During the measurements, the PDs were mounted in a dark, electrically shielded and optically sealed chamber on the optical table to reduce vibrational noise. The spectral photoresponse was detected in the $300-1,100 \mathrm{~nm}$ wavelength range. For this purpose, we used a $450-\mathrm{W}$ xenon light source and a 0.25 -m grating monochromator (Newport Cornerstone $2601 / 4 \mathrm{~m}$ monochromator). The incident light intensity was monitored by a motorized variable wheel attenuator and a Newport calibrated UV-enhanced silicon photodiode. The power density of the incident light on the sample surface was about $1 \sim 320 \mu \mathrm{W} \mathrm{cm}{ }^{-2}$ depending on the wavelength in the range of $300 \sim 1,100 \mathrm{~nm}$. A 532-nm monochromatic light from a diode laser was used for studying the photosensitivity linearity with light power. The light was modulated with a mechanical chopper (Stanford Research Systems) at $30 \mathrm{~Hz}$, and the PC response at various bias voltages was recorded with a Signal Recovery 7,265 DSP lock-in amplifier. Optical neutral density filters were used to vary the light intensity from $\mathrm{nW}$ up to $7.2 \mathrm{~mW}$. (maximum power: $90 \mathrm{~mW}$ ). For frequency dependence of the PC, the graphene $p-n$ PD was illuminated by a Thorlabs $50 \mu \mathrm{W}, 530 \mathrm{~nm}$ light-emitting diode array with an Instek SFG-830 $30 \mathrm{MHz}$ function generator used to supply a constant or modulated bias to the light-emitting diodes. The PC response was measured with an SR530 lock-in amplifier coupled to a current preamplifier (Stanford Research SR570) in the frequency range of $1-100 \mathrm{kHz}$. Transient PC studies were conducted using laser pulses from the Nd:YAG laser (532 nm wavelength, 20 ps duration and $20 \mathrm{~Hz}$ repetition rate, generated by a Continuum Leopard-D20 Nd:YAG laser), which were focused onto the PD devices with a spot size of $\sim 5 \times 5 \mathrm{~mm}^{2}$. A laser power meter (Laser probe, $\mathrm{Rj}-760$ ) was used to measure the average power of the laser pulses. The laser pulse temporal profiles and transient $\mathrm{PC}$ were monitored with the $50 \mathrm{ohm}$ terminated, $500 \mathrm{MHz}$ bandwidth digital oscilloscope (Tektronix DPO 4054). All measurements were performed in a dark, shielded enclosure under vacuum at RT.

\section{References}

1. Zimmermann, H. Integrated Silicon Optoelectronics 29-153 (Springer, 2000).

2. Snyman, L. W. et al. Optical sources, integrated optical detectors and optical waveguides in standard silicon CMOS integrated circuitry. Proc. SPIE 3953 20-36 (2000).

3. Xia, F., Mueller, T., Lin, Y. M., Valdes-Garcia, A. \& Avouris, P. Ultrafast graphene photodetector. Nat. Nanotechnol. 4, 839-843 (2009).

4. Mueller, T., Xia, F. \& Avouris, P. Graphene photodetectors for high-speed optical communications. Nat. Photon. 4, 297-301 (2010)

5. Sun, D. et al. Ultrafast hot-carrier-dominated photocurrent in graphene. Nat. Nanotechnol. 7, 114-118 (2012).

6. Park, J., Ahn, Y. H. \& Ruiz-Vargas, C. Imaging of photocurrent generation and collection in single-layer graphene. Nano Lett. 9, 1742-1746 (2009).

7. Lee, E. J. H., Balasubramanian, K., Weitz, R. T., Burghard, M. \& Kern, K. Contact and edge effects in graphene devices. Nat. Nanotechnol. 3, 486-490 (2008).

8. Xia, F. et al. Photocurrent imaging and efficient photon detection in a graphene transistor. Nano Lett. 9, 1039-1044 (2009).

9. Bonaccorso, F., Sun, Z., Hasan, T. \& Ferrari, A. C. Graphene photonics and optoelectronics. Nat. Photon. 4, 611-622 (2010).

10. Britnell, L. et al. Strong light-matter interactions in heterostructures of atomically thin films. Science 340, 1311-1314 (2013).

11. Vicarelli, L. et al. Graphene field-effect transistors as room-temperature terahertz detectors. Nat. Mater. 11, 865-871 (2012).

12. Xu, X., Gabor, N. M., Alden, J. S., van der Zande, A. M. \& McEuen, P. L. Photothermoelectric effect at a graphene interface junction. Nano Lett. 10, 562-566 (2010).

13. Gabor, N. M. et al. Hot carrier-assisted intrinsic photoresponse in graphene. Science 334, 648-652 (2011).

14. Peters, E. C., Lee, E. J. H., Burghard, M. \& Kern, K. Gate dependent photocurrents at a graphene p-n junction. Appl. Phys. Lett. 97, 193102 (2010)

15. Kim, S. et al. Graphene p-n vertical tunneling diodes. ACS Nano 7, 5168-5174 (2013).

16. Chen, K. et al. Quantitative determination of scattering mechanism in largearea graphene on conventional and SAM-functionalized substrates at room temperature. Nanoscale 5, 5784-5793 (2013).

17. Hwang, E. H., Hu, B. Y. K. \& Das Sarma, S. Inelastic carrier lifetime in graphene. Phys. Rev. B 76, 115434 (2007).

18. Kim, R., Perebeinos, V. \& Avouris, P. Relaxation of optically excited carriers in graphene. Phys. Rev. B 84, 075449 (2011).

19. Winzer, T. \& Malić, E. Impact of Auger processes on carrier dynamics in graphene. Phys. Rev. B 85, 241404 (2012).

20. Song, J. C. W., Rudner, M. S., Marcus, C. M. \& Levitov, L. S. Hot carrier transport and photocurrent response in graphene. Nano Lett. 11, 4688-4692 (2011).

21. Yu, Z., Aceves-Mijares, M., Lopez, J. A. L. \& Deng, J. Nanocrystalline Si-based metal-oxide-semiconductor photodetectors. Proc. SPIE 7381, $73811 \mathrm{H}$ (2009).

22. Shaw, P.-S., Larason, T. C., Gupta, R., Brown, S. W. \& Lykke, K. R. Improved near-infrared spectral responsivity scale. J. Res. Natl Inst. Stand. Technol. 105, 689-700 (2000). 
23. Pal, B. N. et al. High-sensitivity p-n junction photodiodes based on PbS nanocrystal quantum dots. Adv. Funct. Mater. 22, 1741-1748 (2012).

24. Gong, X. et al. High-detectivity polymer photodetectors with spectral response from $300 \mathrm{~nm}$ to $1450 \mathrm{~nm}$. Science 325, 1665-1667 (2009).

25. Konstantatos, G., Levina, L., Tang, J. \& Sargent, E. H. Sensitive solutionprocessed $\mathrm{Bi}_{2} \mathrm{~S}_{3}$ nanocrystalline photodetectors. Nano Lett. 8, 4002-4006 (2008).

26. Manga, K. K. et al. High-performance broadband photodetector using solutionprocessible $\mathrm{PbSe}_{\mathrm{TiO}}$-graphene hybrids. Adv. Mater. 24, 1697-1702 (2012).

27. Gao, L.-P., Wei, J., Wang, Y.-C., Ding, G.-J. \& Yang, Y.-L. A new repeatable, optical writing and electrical erasing device based on photochromism and electrochromism of viologen. Smart Mater. Struct. 21, 085006 (2012).

28. Konstantatos, G. \& Sargent, E. H. Nanostructured materials for photon detection. Nat. Nanotechnol. 5, 391-400 (2010).

\section{Acknowledgements}

We are grateful to Professor Philip Kim at Columbia University for his helpful discussions. This work was supported by Basic Science Research Program through the National Research Foundation of Korea (NRF) funded by the Ministry of Science, ICT and Future Planning (Number 2011-0017373).

\section{Author contributions}

J.S.L. and J.H.K. deposited graphene sheets by CVD. C.W.J. and J.M.K. prepared doped graphene sheets. S.K. structurally characterized the doped graphene. S.S.K. carried out optical measurements. D.H.S. and S.K. fabricated graphene $p-n$ junctions. C.O.K. characterized performances of PDs together with S.S.J. and E.H. contributed to the theoretical interpretation of the results. S.-H.C. initiated, supervised the work and wrote the paper. All authors discussed the results and commented on the manuscript.

\section{Additional information}

Supplementary Information accompanies this paper at http://www.nature.com/ naturecommunications

Competing financial interests: S.-H.C., S.K. and C.O.K. have a Korea patent pending (10-2013-0003539) on the graphene $p-n$ PD production technology, reported in this paper.

Reprints and permission information is available online at http://npg.nature.com/ reprintsandpermissions/

How to cite this article: Kim, C. O. et al. High photoresponsivity in an all-graphene $p-n$ vertical junction photodetector. Nat. Commun. 5:3249 doi: 10.1038/ncomms4249 (2014). 